\title{
Refractory Trigeminal Neuralgia: Successful Treatment with LINAC Based Stereotactic Radiosurgery (SRS): First Case in Bangladesh
}

\author{
Taohida Yasmin ${ }^{1}$, Narendra Kumar ${ }^{2 \#}$ Sandip K Das ${ }^{3}$, Murugan Appasamy ${ }^{4}$, K M Masud Rana ${ }^{5}$, \\ Porama Zafreen ${ }^{6}$, R. Arun Kumar ${ }^{7}$, Sania Ahsan,
}

Conflict of interest: There is no conflict of interest relevant to this paper to disclose.

Funding Agency: was not funded by any institute or any group.

Contribution of Authors: None

Copyright: @2020bang.BJNS published by BSNS. This article is published under the creative commons CC-BY-NC license. This license permits use distribution (https://creativecommons. orgf/licences/by-nc/4-0/)reproduction in any medium, provided the original work is properly cited and is not used for commercial purposes.

Received: 07.07.19

Accepted: 21.10 .19

\begin{abstract}
:
Purpose: To present first case of refractory trigeminal Neuralgia treated with SRS in Bangladesh, procedural technique, and outcomes in terms of pain relief.

Background: Trigeminal neuralgia (TN), classically known as tic doloureaux is a chronic and recurrent disabling pain syndrome, which described as episodes of lancinating pain over the face along the sensory distribution of trigeminal nerve. First line management of TN is medical with different permutation \& combination to control the pain. After the failure of medical management, non-invasive SRS is an established modality to achieve long term pain control. Here, we are reporting a case of TN treated with LINAC based SRS.

Case Presentation: A 61 years old, gentleman who developed piercing pain inside his left eye for a duration 1-1.5 sec, precipitated while shaving, brushing teeth in year 2015, occurred 4-5 time a day. He was diagnosed as left TN of V1, started on Carbamazepine, Pregabalin. In 3 years, pain progressed to involve all 3 branches. Even combination Carbamazepine, Gabapentin, Tramadol, Amitriptyline, Clonazepam, $\&$ Morphine could not control the pain. Pain was persisting all over the day and he also developed suicidal tendency. Later he has been referred to us for SRS. SRS was done in April-2019, a dose of 90Gy was delivered to the Distal Retrogasserian (RG) also called Marseille point of trigeminal nerve root. Eight months after the SRS patient is almost free of pain without any Medicine.
\end{abstract}

Conclusions: LINAC based SRS is a non-invasive, frameless, and safe procedure with excellent pain control for refractory Trigeminal neuralgia.

Key words: Trigeminal Neuralgia, Stereotactic radiosurgery, Linac based SRS, Frameless Radiosurgery.

Bang. J Neurosurgery 2020; 10(1): 123-129

\section{Introduction:}

Trigeminal neuralgia (TN) is a neurologic syndrome that is characterized by sudden, severe, brief and recurrent episodes of pain along the trigeminal nerve dermatomes, which is described to be stabbing or electric shock like sensation and usually involves maxillary branch ${ }^{1}$. Pain is triggered by cutaneous stimulations and facial movement like brushing teeth, shaving, chewing drinking, a light touch or even a cool breeze $^{2}$. The annual incidence of Trigeminal neuralgia is 12.6 per 100,000 having male female ratio $2: 3$. Peak age of diagnosis is 60-70 years, and it is uncommon

1. Registrar, Department of Radiation Oncology, Cancer Care Centre, Evercare Hospital Dhaka,

2. Consultant and head of department, Department of Radiation Oncology, Cancer Care Centre, Evercare Hospital Dhaka

3. Consultant Neurologist, Department of Neurology, Evercare Hospital Dhaka,

4. Chief medical physicist, Department of Radiation Oncology, Cancer Care Centre, Evercare Hospital Dhaka,

5. Medical physicist, Department of Radiation Oncology, Cancer Care Centre, Evercare Hospital Dhaka,

6. Medical officer, Department of Radiation Oncology, Cancer Care Centre, Evercare Hospital Dhaka,

7. Chief technologist, Department of Radiation Oncology, Cancer Care Centre, Evercare Hospital Dhaka,

8. Consultant, Department of Neuroradiology, Evercare Hospital Dhaka,

Address of Correspondence: Prof. Dr Narendra Kumar, Consultant \& Head, Department of Radiation Oncology, Evercare Hospital Dhaka -1229 , Bangladesh, Phone: +8801777763535, Email: narendra.kumar@evercarebd.com, drnarendra74@gmail.com 
before 40 years $^{3}$. According to International Classification of Headache Disorder (The International Headache Society Criteria, 3rd edition 2014), there are two subtypes of trigeminal neuralgia. First the classic form (80-90\%) of TN with no apparent cause other than neurovascular compression of trigeminal nerve while taking exit from the brain stem by aberrant arterial or venous loop, most commonly superior cerebellar artery. Second The non-classical form (5$10 \%)$ represents trigeminal neuropathies due to multiple sclerosis or secondary to space occupying lesion in cerebello-pontine angle ${ }^{4}$. Third less used type is Idiopathic TN without any apparent cause. ${ }^{9}$

TN occurs due to chronic compression or injury or inflammation to the trigeminal nerve by vascular malformation, secondary structural disease or any underlying inflammatory condition. The compression of trigeminal nerve causes demyelination followed by dysmyelination, which is remyelination with persistent abnormalities. It is commonly hypothesized that in TN, high frequency ectopic impulses are either generated from or augmented by the areas of dysmyelination ${ }^{5}$. These abnormal discharges might ignite a chain reaction of neuronal depolarization in the trigeminal ganglion ${ }^{6}$. These cascades of neuronal activity are propagated centrally into trigeminal nucleus which is perceived by the patient as an overwhelming burst of pain.

Typical primary treatment strategies for the electric shock-like or stabbing pain of TN is pharmacotherapy with Voltage-gated, frequency-dependent sodium channel blockers. To state in a simple way, medical management is the first step of approach ${ }^{7}$. According to the American Academy of Neurology (AAN), and the European Federation of Neurological Societies (EFNS) this antiepileptic drug, in particular carbamazepine (CBZ), oxcarbazepine (OXC) is the first line of choice. For patients who are initial responder or non- responder for CBZ or OXC, baclofen, lamotrigine is suggested as add-on or second line. Levetiracetam, Gabapentin, pregabalin, topiramate is challenged as third line approach ${ }^{8}$.

Refractory TN is defined as those TN patients, who despite taking at least 3 drug trials with dosage adequate for most patients with adequate pain relief, fails to achieve desired pain relief. (Also called nonresponder). For refractory trigeminal neuralgia or when medical management associated with significant side effects, surgical interventions such as microvascular decompression, ablative procedure like Rhizotomy on the Gasserian ganglion is next option ${ }^{9}$. For medically or surgically refractory $\mathrm{TN}$ or patients who are not eligible for surgeries due to advanced age or presence of medical co-morbidities, Stereotactic radiosurgery (SRS) is the standard of care of treatment. Until the invention of LINAC based SRS, Gamma knife based radiosurgery (GKRS) was solely being used. GKRS unit, in which $201{ }^{60} \mathrm{Co}$ sources are arranged on a spherical helmet with beam diameter 1 to $4 \mathrm{~mm}$ to deliver the desired dose 7,10 . GKRS is an outpatient procedure in which a multiple high dose radiation beam is focused at the trigeminal root entry zone to deliver radiation on a single exposure. This high dose singe fraction radiation over time causes axonal degeneration and necrosis. This interrupts ephaptic transmission as well as the physiological axonal conduction which results in pain relief and loss of facial sensation ${ }^{11}$. Development of Linac based SRS is now gaining appreciable acknowledgement because of its capability of targeting small lesions \& deliver precise radiation in a faster, safer and effective fashion by virtue of its technological advancements without any difference in outcome ${ }^{12}$

Here, we are reporting our $1^{\text {st }}$ case of TN treated with Linac based stereotactic radiosurgery with the objective to describe the technique of SRS delivery and outcomes, which is first case in entire Bangladesh.

\section{Case report:}

Clinical scenario:

Index case is a 61-year old, gentleman man, without any comorbidity, presented with having stabbing pain in his left eye in 2015, for a period of 1-1.5 seconds only, pain was precipitated by shaving, brushing teeth and occasionally spontaneously. Initially the pain was infrequent, having bouts for 4-5times a day without radiation. He was seen by ophthalmologist first. Later sent to neurology consultation. He came to department of neurology at Apollo Hospitals Dhaka in Oct, 2015. Brain MRI on $8^{\text {th }}$ Oct 2015 Found small vessel from left posterior cerebral artery was abutting left trigeminal nerve root entry zone. Eventually was diagnosed to have left V1 Trigeminal neuralgia. He was started with Carbamazepine (100mg every twelve hours), Pregabalin (50 mg every eight hours). He was on regular follow-up with different combination of medicine. He never discontinued the medicines in last four year. In 2019 neuralgic pain spread to all V1, V2 and V3 distribution of trigeminal nerve. He was started on more combination 
of drug with escalation of dose i.e. Carbamazepin (400mg every eight hours), Gabapentin (300mg every eight hours), Piracetum (800mg every 12hours)Tramadol (50mg every eight hours), Morphin (15mg every eight hours), Amitryptillin $25 \mathrm{mg}$, Clonazepum $0.5 \mathrm{mg}$ daily. His pain was aggravated to such extent that he describes his pain to be suicidal, constant remaining almost all day. He was unable to sleep even. Patient was in complete refusal state to perform any clinical examination. He could hardly maintain oral hygiene. He had been offered surgical treatment but refused to undergo any surgical ablative procedure. Subsequently he was referred to department of Radiation oncology in April-2019. After taking informed written consent explaining the benefits, complications, outcome and the consequences of the treatment. He was treated with stereotactic Radiosurgery on 21.4.2019 with a dose of $90 \mathrm{~Gy}$ delivered to Distal Retrogasserian (RG) also called Marseille point of trigeminal nerve root. On his last follow up after 10-months post treatment, he was found to have $90 \%$ improvement in Pain. He is pain free.

\section{Procedural technique:}

After obtaining informed written consent, he was taken up for preparation of the treatment. Initially the patient was positioned on a special head support (Elekta Fraxion), which can be customized depending upon patient's comfort then a double layer (Elekta Fraxion) thermoplastic mask with three clamps is used to position the patient with chin up position.

After this the patient is shifted to CT scanner (GE, Discover VCT) to acquire axial CT image of patient $1 \mathrm{~mm}$ thin slices with contrast with stereotactic localizer box in situ (as shown in figure 1). The localizer box has " $Z$ " shaped markers (Figure 1) around the patients (laterals

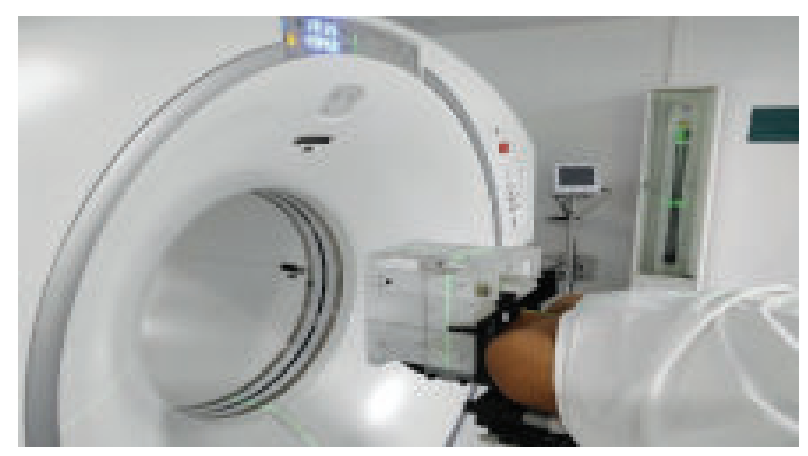

Fig.-1: Stereotactic Localizer Box
And anterior) to give nine localizing markers in axial slices, which helps us to locate the stereotactic coordinates of tumor with reference to Isocentre of treatment plan. The whole process is done with submillimetric accuracy.

Later high-resolution magnetic resonance imaging (MRI) with contrast consists of $1 \mathrm{~mm}$ thin slices with T1-weighted, T2-weighted images was acquired in the same set up for treatment position. MRI was coregistered with the planning $C T$ image set as shown in figure 3.

Radiation oncologist and Neuro-radiologist delineated the distal Retrogasserian (RG) also called Marseille point of trigeminal nerve root. Organs at risk (OAR) were delineated on the co-registered MRI image that consisted brainstem, temporal lobe, optic apparatus, Cochlear apparatus etc. The radiosurgery team of Radiation oncologist, neurosurgeon and medical physicist, with the intension of avulsion of trigeminal nerve (Target Volume $0.109 \mathrm{~cm}^{3}$ ), prescribed a dose of $9000 \mathrm{cGy}$ for in single exposure. After contouring, The SRS treatment plan was generated with the help of Monaco (Elekta, Version 5.3) treatment planning System with the intention to deliver maximum dose to the target and minimizing the dose to all OARs. For this Planning, VMAT inverse treatment technique is used with 8 non-coplanar partial arcs as shown in figure-4.

The treatment plan was evaluated carefully against the single fraction RTOG OARs constraints by both medical physicist and radiation oncologist by analyzing the Dose Volume Histogram (Figure 5) before approving it for treatment.

Figure 5: showing isodose distribution (color-wash) in three sections (axial, coronal \& sagittal), Dose Volume Histogram (DVH) of the patient.

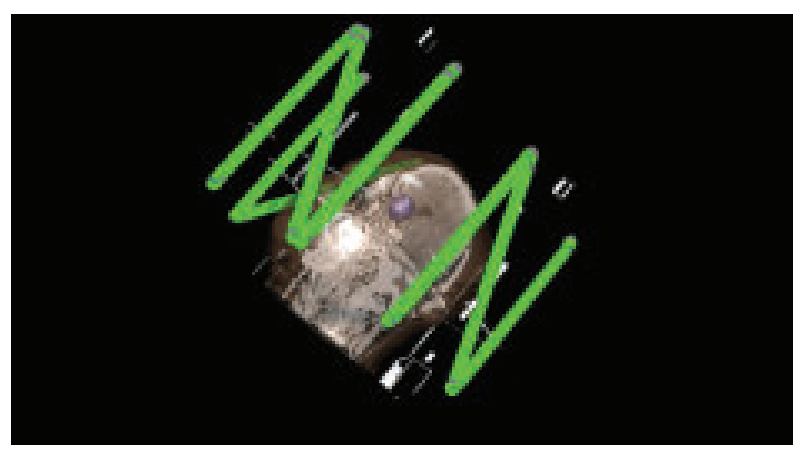

Fig.-2: Showing "Z" shaped marker to locate tumor with Stereotactic co-ordinates 
Once the plan is approved for treatment, a comprehensive patient specific Quality assurance (QA) was carried out to assure that the intended dose would be delivered accurately within acceptable limits. It includes, collision check, dummy run and dosimetric fluence verification with the help of Imatrix (IBA, Germeny). In this dosimetric verification, the plan was delivered to a phantom and the $2 \mathrm{D}$ fluence of the measured dose is evaluated against TPS calculated fluence. The passing result was found to be more than $98 \%$ for this case. Normally any plan with passing rate more than $95 \%$ is suitable for treatment delivery. After the Patient specific QA, the patient was scheduled for treatment.

During the treatment, reproducibility of the patient position was verified using KVCT \& hexapod $6 \mathrm{D}$ couch prior to the radiation delivery to ensure $\pm 1 \mathrm{~mm}$ accuracy (As shown in figure 6) and precision of treatment. Later treatment was delivered by Versa HD Treatment unit using SRS Technique using 6.0 MV FFF photon energy.
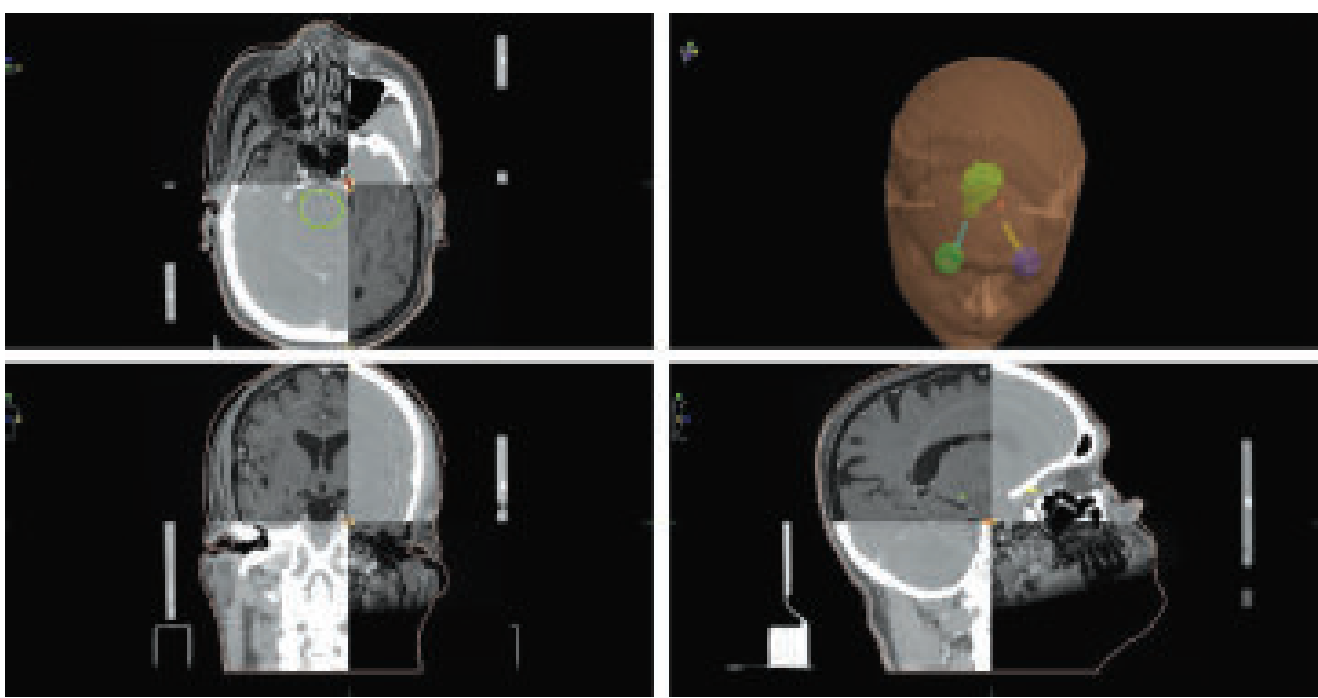

Fig.-3: Showing the fusion (Co-Registration) of MRI images with CT for delineation of target
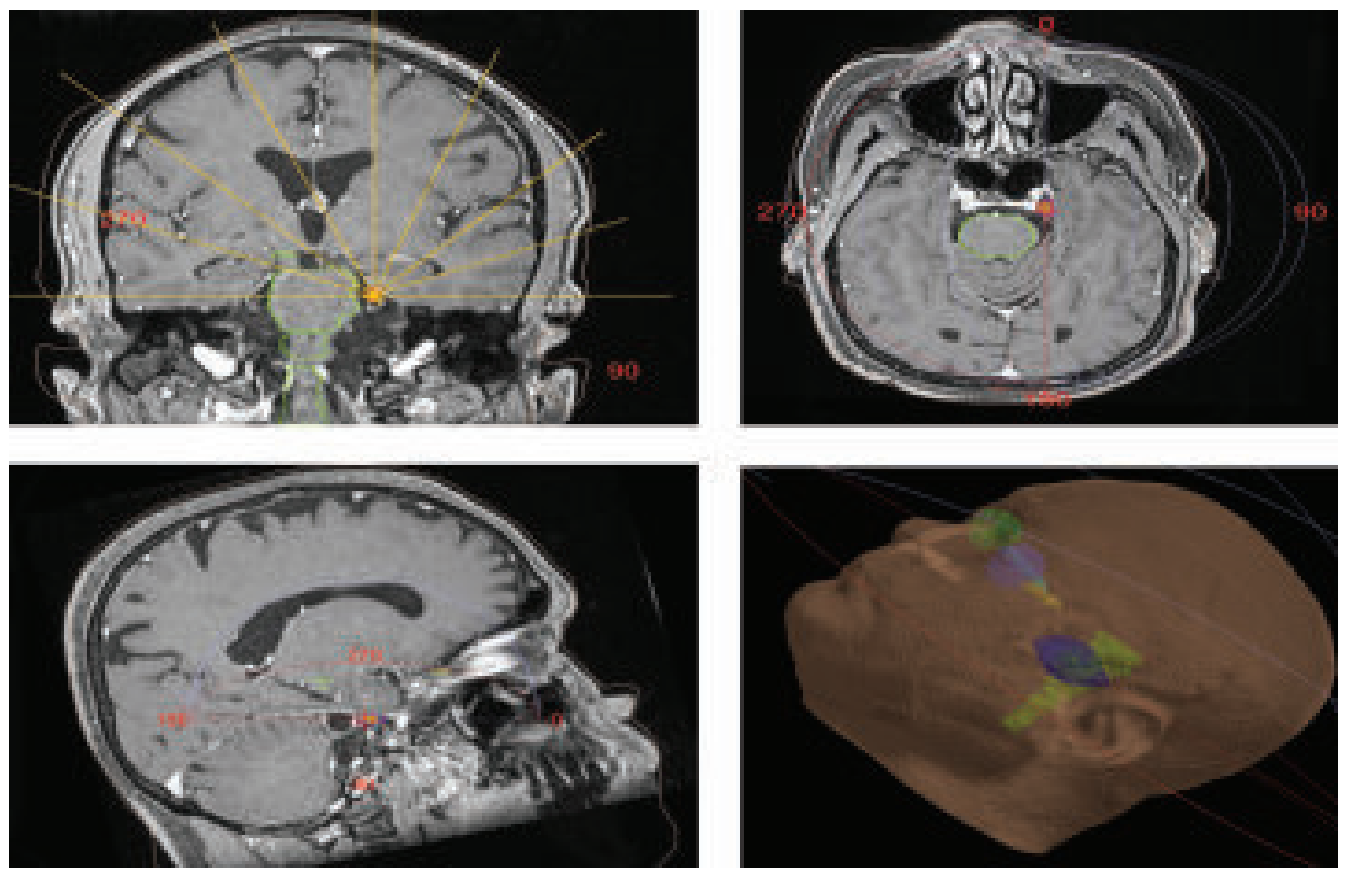

Fig.-4: Figure showing 8 non-coplanar arcs used for this patient 

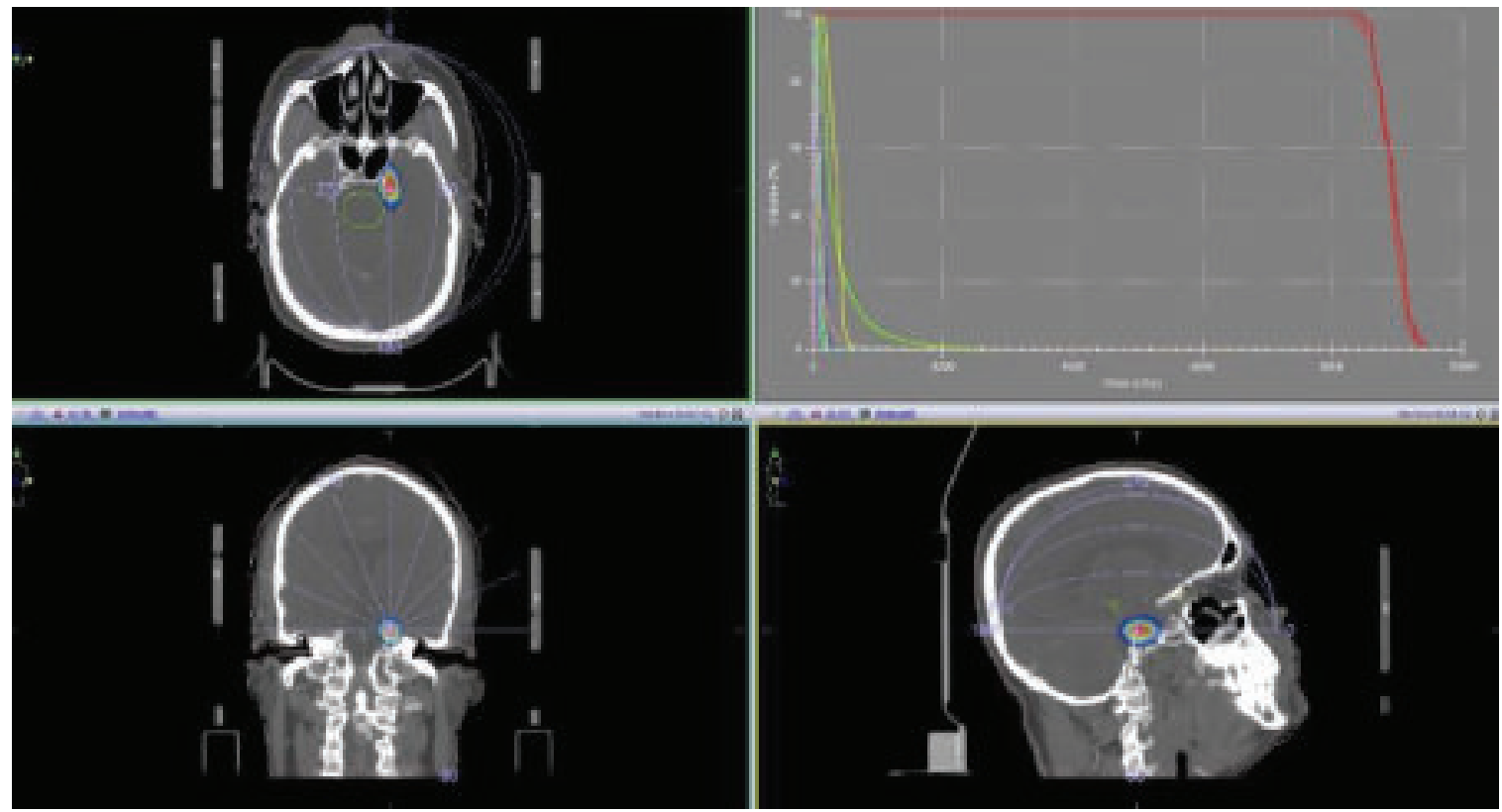

Fig.-5: Showing isodose distribution (color-wash) in three sections (axial, coronal \& sagittal), Dose Volume Histogram (DVH) of the patient.

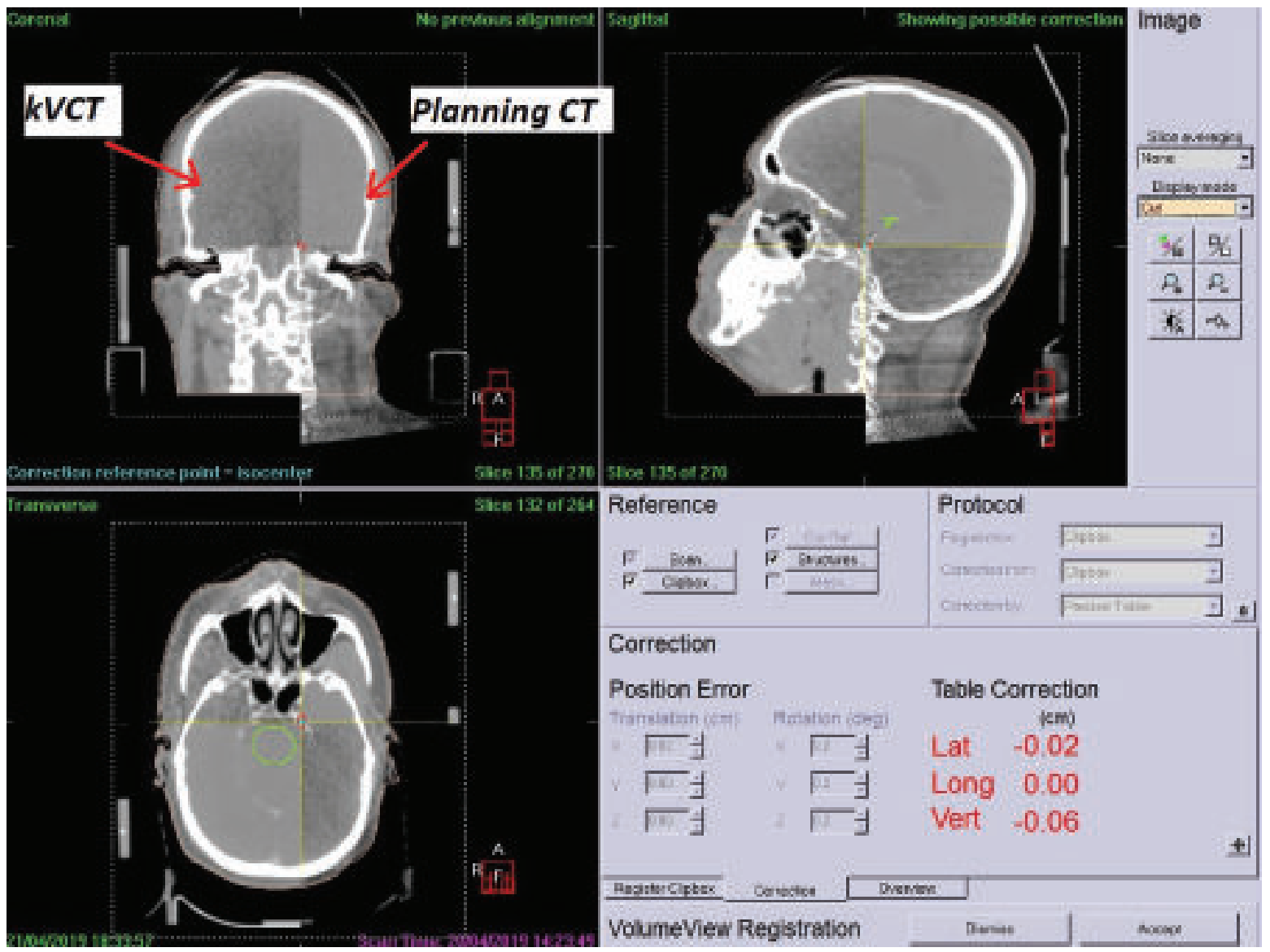

Fig.-6: Showing the Co-axial registration of onboard kVCT obtained just before treatment with planning CT for verifying the accuracy of patient positioning and the maximum shift was found to be less than $1 \mathrm{~mm}$ 
Outcome: Our primary outcome was "Pain relief". Before the SRS, His Pain intensity according to Barrow neurological Institute (BNI) scoring criteria was V. He was pain free within 10 days post procedure. Despite, He was advised to continue the medications after surgery for two weeks with unchanged dosage. His medication was tapered gradually over a period of 12 weeks in his last follow up in February'20, pain score was I with no medications.

Apart from loss of corneal reflexes and Facial numbness with BNI score III, which was expected, he was free of new complains of headache, cranial nerve deficit, focal neurological deficit or seizure activity.

\section{Discussion:}

trigeminal neuralgia, also known as "tic doloroux" is an extremely painful condition characterize by chronic recurrent disabling pain along the sensory distribution of Cranial nerve V. Diagnosis is essentially clinical. Imaging studies are important to discriminate the type of neuralgia, evaluation of atypical neuralgia. This pain syndrome is responsive to medical and surgical therapies. For refractory neuralgia, radiosurgery has been an excellent treatment of choice. In this case report our patient was refractory to the Medical management. Options for surgical intervention was deferred by patient. He opted to go for a noninvasive procedure.

The anatomical target for radiation in this patient with a prescribed dose was 90Gy, was distal retrogasserian portion of Trigeminal nerve root, merseille point (distalmost point of cisternal component of trigeminal nerve close to Meckel's cave) to reduce radiation exposure of the brainstem. In 2001, Regis et al ${ }^{13}$ published a study in which 53 patients were treated with GKS and evaluated different radiosurgical approach. Authors encouraged a more anterior radiosurgery target for TN coupled with 90 Gy to achieve pain control with lower morbidity. Massager et al. ${ }^{14}$, suggested to keep the target volume of nerve 5 to $8 \mathrm{~mm}$ distant from brainstem for an optimal balance between better pain control utilizing higher dose and limited adverse effects. Kondzio lka et al. while in 1996 concluded that the proximal trigeminal nerve and the root entry zone was the most appropriate target in surgery ${ }^{15}$, later in 1998 they advocated to keep the target volume 2 to $3 \mathrm{~mm}$ anterior to the REZ to minimize the radiation exposure to brainstem ${ }^{16}$.
He had improvement of pain within 24 hours of the surgery, this supports the literature by Smith ZA et $\mathrm{al}^{11}$ who published single center experience on 179 TN patients with D-LINAC with different dose prescriptions and treatment plans. They described that time to initial pain relief and was notably shorter ( 0 months vs 3.0 months) and the durability and sustainability of pain relief at 1 year was significantly higher $(p=0.002)$ for $90-\mathrm{Gy}$ treatment than 70-Gy. Regis et al ${ }^{13}$ reported that $87 \%$ Patient had pain control with 90 Gy prescription dose.

Patient continued drug treatment for about 4 weeks post-surgery and had $80 \%$ relief of pain within a month. Later the medications were tapered off gradually over period of time. In his last follow up which is about 8 month later, he is completely free of pain no medications. Published data of Kondzio lka et al. in his study supports the median time to pain relief was 01 month $^{15}$.

\section{Conclusion:}

The Stereotactic radiosurgery delivered to the patient was found to have excellent pain control for Refractory TN. On follow up at 8 months post-surgery, Patient was clinically stable with no pain \& no medicine. This patient report and further follow-up suggest that, the Linear accelerator based Stereotactic Radio Surgery can be effectively utilized for the management of Trigeminal Neuralgia, in places where a dedicated facility is not available, and the existing Linear Accelerator can be used with additional accessories.

\section{References:}

1. Benetto L, Patel NK, Fuller G - Trigeminal neuralgia and its management. BMJ 2007; 334:201-205

2. Krafft RM - Trigeminal neuralgia. Am Fam Physician 2008; May1;77(9): 1291-6

3. Koopman JS, Dieleman JP, Huygen FJ, de Mos M, Martin $\mathrm{CG}$, Sturkenboom MC. Incidence of facial pain in the general population. Pain. 2009; 147:122-7

4. Headache Classification Committee of the International Headache Society (IHS). The international classification of headache disorders, $3^{\text {rd }}$ edition. Cephalgia. 2018:38:1211.

5. Bruchiel KJ. Abnormal impulse generation in focally demyelinated trigeminal roots. J Neurosurg. Nov 1980;53(5):674-83.

6. Devor M, Amir R, Rappaport ZH. Pathophysiology of trigeminal neuralgia: the ignition hypothesis. Clin J Pain. Jan-Feb 2002;18(1):4-13

7. Tripathi M. Trigerminal neuralgia: An orphan with many fathers. Nerol India 2019;67:414-6 
8. Cruccu G, Gronseth G, Alksne J, Argoff C, Brainin M, Burchiel $\mathrm{K}$, et al. AAN-EFNS guidelines on trigeminal neuralgia management. Eur J Neurol 2008; 15: 1013-1028.

9. Han I, Shin D, Chang J, Kim K, Chnag J,Huh R, Chung S. effect of Various Surgica Modalities in recurrent or persistent trigeminal neuralgia. Sterotactic Funct Neurosurg. 2010; 88:156-62.

10. Kondziolka D, Zorro O, Lobato-Polo J, et al. Gamma Knife Stereotactic Radiosurgery for idiopathic trigeminal neuralgia. J Neurosugery. 2010; 112:758-765.

11. Sheehan J, Pan HC, Stroila M, Steiner L. Gamma knife surgery for trigeminal neuralgia: outcomes and prognostic factors. J Neurosurg 2005; 102: 434-441.

12. Smith ZA, Gorgulho AA, Bezrukiy N, et al. Dedicated linear accelerator radiosurgery for trigeminal neuralgia: A single center experience in 179 patients with varied dose prescriptions and treatment plans. Int J Radiat Biol Phys. 2011; 81:225-231.

13. Regis J, Metellus $\mathrm{P}$, Dufour $\mathrm{H}$, Roche $\mathrm{P}$, Muracciole $\mathrm{X}$ et al. Long-term outcome after gamma knife surgery for secondary trigeminal neuralgia. J Neurosurgery 2001; 95:199-205

14. Massager N, Lorenzoniu J, Devriendt D, et al. Gamma knife surgery for idiopathic trigeminal neuralgia performed using far-anterior cisternal target and high dose of radiation. J Neurosurgery 2004; 100: 597-605

15. Kondziolka D, Lunsford LD, Flickinger JC, et al. Stereotactic radiosurgery for trigeminal neuralgia: a multi-institutional study using gamma unit. J Neurosurg. jun 1996:84(6):940-5

16. Kondziolka D, Perez B, Flickinger JC et al. Gamma knife radiosurgery for trigeminal neuralgia: results and expectation. Arc Neurol. Dec 1998;55(12):1524-9 